\title{
Acute Mesenteric Ischemia Complicated with Pneumoperitoneum, Pneumoporta, and Pneumatosis Intestinalis
}

\author{
Cheng-Kuo Chen, Chun-Fu Ting, Chin-Yuan Yii and Jen-Wei Chou
}

Key words: acute mesenteric ischemia, pneumoperitoneum, pneumoporta, pneumomatosis intestinalis

(Intern Med 50: 1351, 2011)

(DOI: 10.2169/internalmedicine.50.5477)

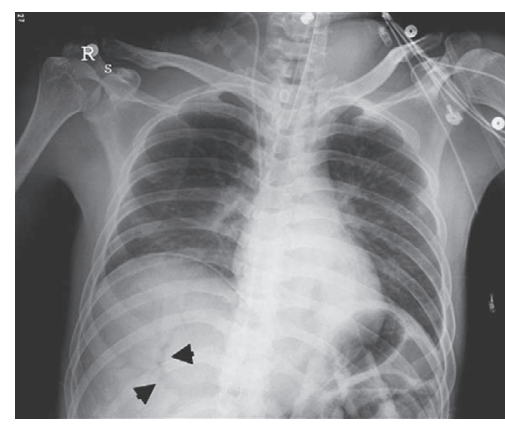

Picture 1.

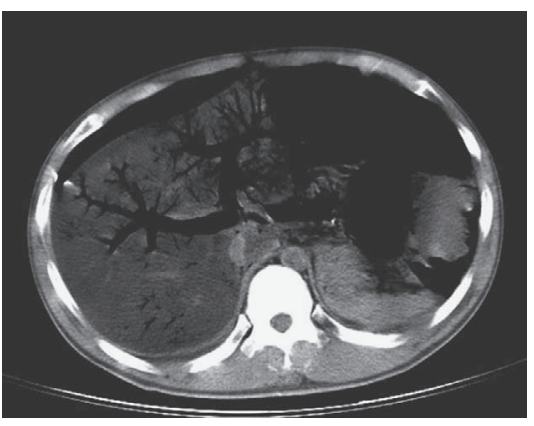

Picture 2.

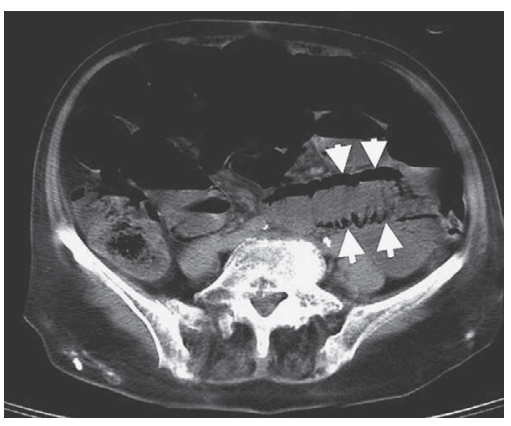

Picture 3.
A 31-year-old man with known alcoholism presented to our hospital because of epigastric pain. Acute pancreatitis was diagnosed and intravenous hydration was given. However, he drank alcohol despite having been given advice not to drink. On admission day 10, multiple abrasions and ecchymoses were found on his skin when he returned to the clinic. Subsequently, he experienced severe abdominal pain, hypotension, and loss of consciousness. Resuscitation with emergency intubation was performed. A portable chest radiograph showed air with tree-like distribution in the right upper abdomen, suggestive of the pneumoporta (Picture 1, arrows). Abdominal computed tomography (CT) demonstrated air in the peritoneum, the portal system, and the mesenteric veins (Picture 2). Moreover, air in the small bowel wall was identified (Picture 3, arrows). Acute mesenteric ischemia (AMI) complicated with pneumoperitoneum, pneumoporta, and pneumatosis intestinalis was diagnosed. Surgical intervention was not indicated because of multiple organ failure. The patient died two days later.

AMI is a syndrome in which inadequate blood flow through the mesenteric circulation causes ischemia and eventual gangrene of the bowel wall. It is divided into 4 subtypes: acute mesenteric arterial embolus, acute mesenteric arterial thrombosis, non-occlusive mesenteric ischemia, and mesenteric venous thrombosis (1). Multidetector CT has a sensitivity of $93.3 \%$ and a specificity of 95.9 for AMI (2). It is ordered much more frequently than conventional angiography because of its noninvasive and readily available characteristics. The overall mortality rate of AMI averages $71 \%$, with a range of $59-93 \%$. Once bowel wall infarction has occurred, the mortality rate is as high as $90 \%$.

The authors state that they have no Conflict of Interest (COI).

\section{References}

1. Lock G. Acute mesenteric ischemia: classification, evaluation and therapy. Acta Gastroenterol Belg 65: 220-225, 2002.

2. Menke J. Diagnostic accuracy of multidetector CT in acute mesenteric ischemia: systematic review and meta-analysis. Radiology 256: 93-101, 2010.

Division of Gastroenterology and Hepatology, Department of Internal Medicine, China Medical University Hospital, China Medical University, Taiwan

Received for publication March 11, 2011; Accepted for publication March 14, 2011

Correspondence to Dr. Jen-Wei Chou, codecol@yahoo.com.tw

(C) 2011 The Japanese Society of Internal Medicine Journal Website: http://www.naika.or.jp/imindex.html 